\title{
CHEST WALL MASS - IS IT TB OR NOT TB?
}

\section{Sonal Kapoor ${ }^{1}$, Hema Gandecha ${ }^{1}$, Srini Bandi ${ }^{1}$}

${ }^{1}$ Leicester Children's Hospital, University Hospitals of Leicester NHS Trust, Leicester, UK

\section{Background}

Musculoskeletal tuberculosis (TB) accounts for $1-3 \%$ of patients with active disease However cold abscess of the chest wall is rare $(1 \%$ to $5 \%$ of cases of all musculoskeletal TB). About $1 / 3^{\text {rd }}$ of children with TB will have extrapulmonary manifestations. Patients are often young and with no comorbidity. Diagnosis is often delayed because of the atypical presentation and lack of awareness. We describe here a case of anterior chest wall mass as the presenting feature of tuberculosis.

\section{Case}

A 15 year old girl, Bangladeshi in origin presented with a 2 month history of a mass on the left anterior chest wall. She had a cough and intermittent fever for 2 weeks. There were no other systemic symptoms. She received BCG at birth. On examination she had a $6 \mathrm{~cm} \times 4 \mathrm{~cm}$ mass overlying the left 8th and 9th ribs. Chest x-ray and a subsequent MRI showed the mass eroding into the underlying 8th rib, hilar adenopathy and a small left pleural effusion. The patient underwent a diagnostic biopsy to exclude malignancy. This revealed a fluid filled centre and a significant amount of pus was drained. A post- biopsy chest $x$ ray showed a markedly enlarged pleural effusion. The patient's IGRA (QuantiFERON) test was positive $(16.05 \mathrm{lU} / \mathrm{ml})$. The pleural fluid was smear negative and the culture confirmed drug sensitive Mycobacterium tuberculosis. The patient was treated with standard AntiTubercular Therapy and had an uneventful recovery.

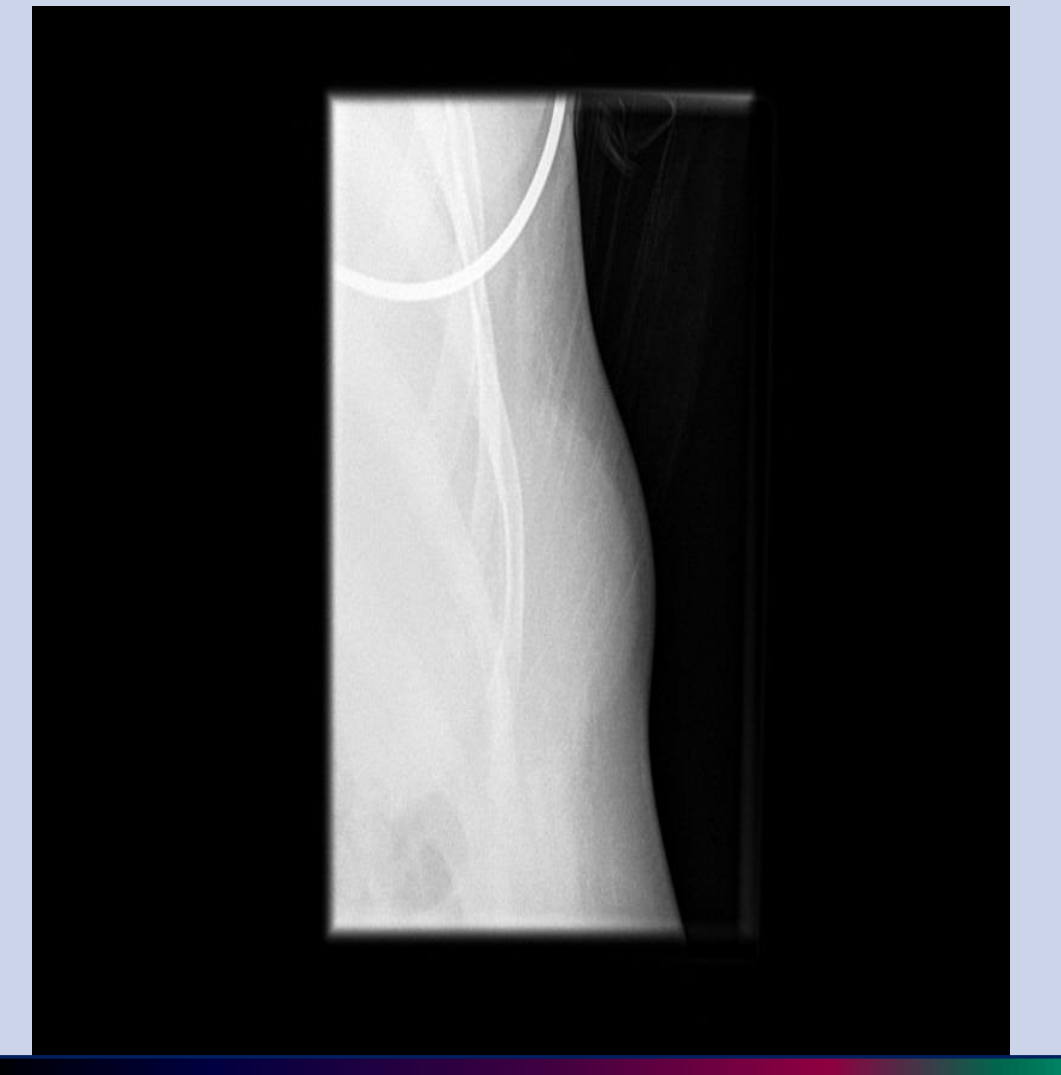

Pre-treatment CXR \& MRI

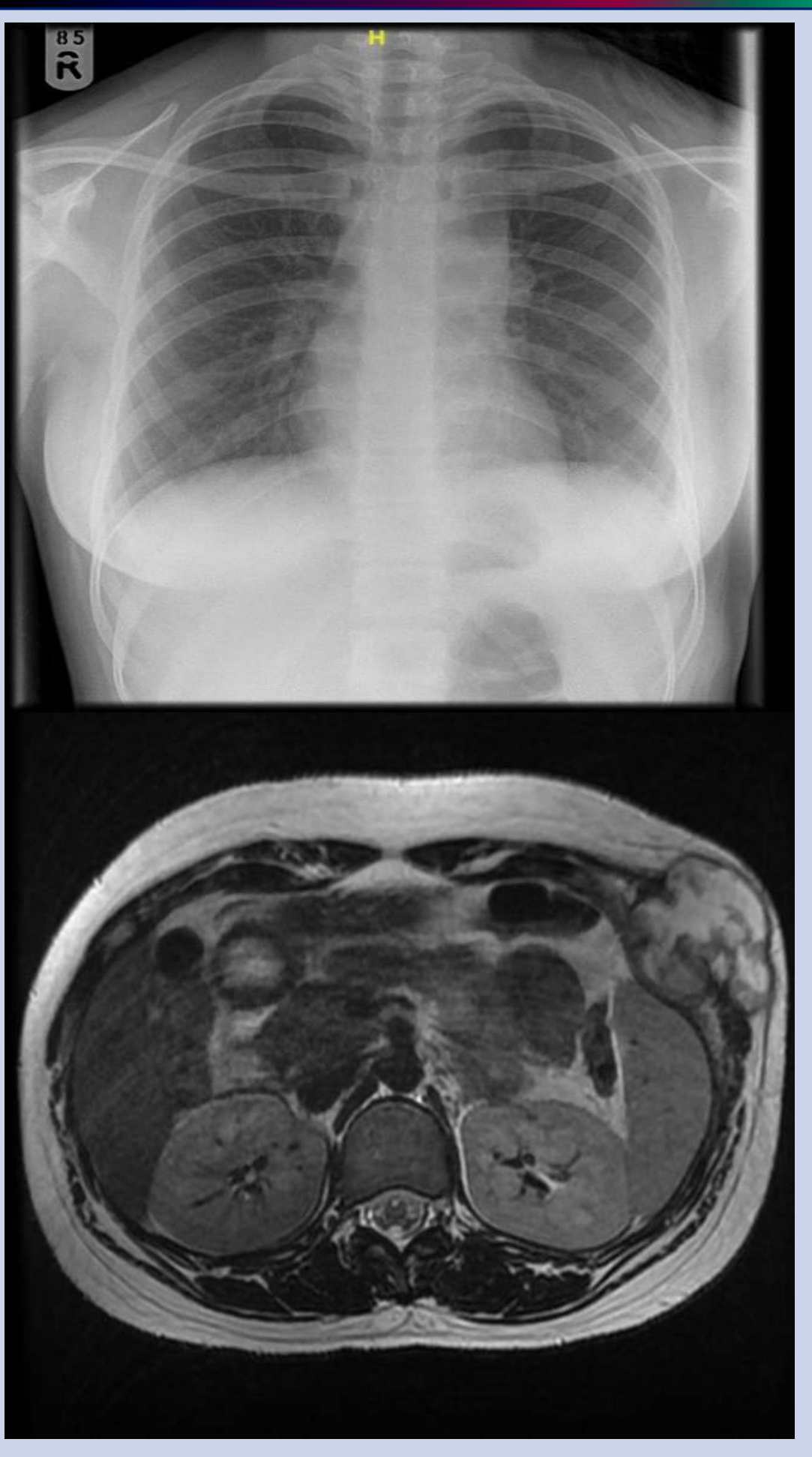

\section{Discussion}

Leicester has one of the highest rates of TB (38.5 per 100,000) in England. In 2016, (in the East Midlands) TB incidence rate was 21 times higher in those born outside the UK (48.2 cases per $100,000)$ compared to the UK born population (2.3 cases per 100,000). The number of cases and the incidence rate of TB in the non-UK born population have seen a year- on-year decline since 2012

Skeletal TB occurs primarily in the first three decades of life. The characteristics are insidious, monoarticular onset with constitutional symptoms along with local signs and symptoms of bone involvement. The most common site of involvement is spine. A marked, exudative reaction is common in tuberculous infection of the skeletal system. A cold abscess is commonly see and may penetrate the ligaments and periosteum. A superficial abscess may burst to form a sinus or an ulcer. On aspiration, the contents of the cold abscess may range from serous fluid to thick purulent pus. Following infection, marked hyperemia and severe osteoporosis can take place. Depending upon the sensitivity pattern, the host immunity and the stage of lesion at the inception of treatment, the tuberculous lesion may behave in a variety of patterns. It can resolve completely (as in our case), may heal with residual deformity and loss of function, be completely walled off and calcify, persist as low grade chronic fibromatous granulomatous lesion or may spread locally.

References:

1.llija Kirovski. Anterior chest wall tuberculosis in children. European Respiratory Journal 2015; 46: PA2766 2.Bahadir Ayse, Mediha Gönenc Ortaköylü, Levent Cansever, Belm.Akbaba, Mehmet Ali Bedirhan. Primary sternal tuberculosis mimicking anterior chest wall tumor: A case report. European Respiratory Journal 2015 46: PA2761

3.Held MFG, Hoppe S, Laubscher M, Mears S, Dix-Peek S, Zar HJ, Dunn RN. Epidemiology of Musculoskeletal Tuberculosis in an Area with High Disease Prevalence. Asian Spine J. 2017 Jun;11(3):405-411

4. Hsu HS, Wang LS, Wu YC, Fahn HJ, Huang MH. Management of primary chest wall tuberculosis. Scand J Thorac Cardiovasc Surg. 1995;29(3):119-23.

University Hospitals of Leicester WHS NHS Trust

\section{Conclusion}

Tuberculosis is a great masquerader and atypical presentations are known to occur. TB should be considered in children and young people presenting with a chest wall mass with hilar adenopathy. Other differential diagnoses include benign and malignant tumours. Prompt diagnosis and treatment results in complete recovery and helps in preventing serious complications.

\section{Post treatment CXR}

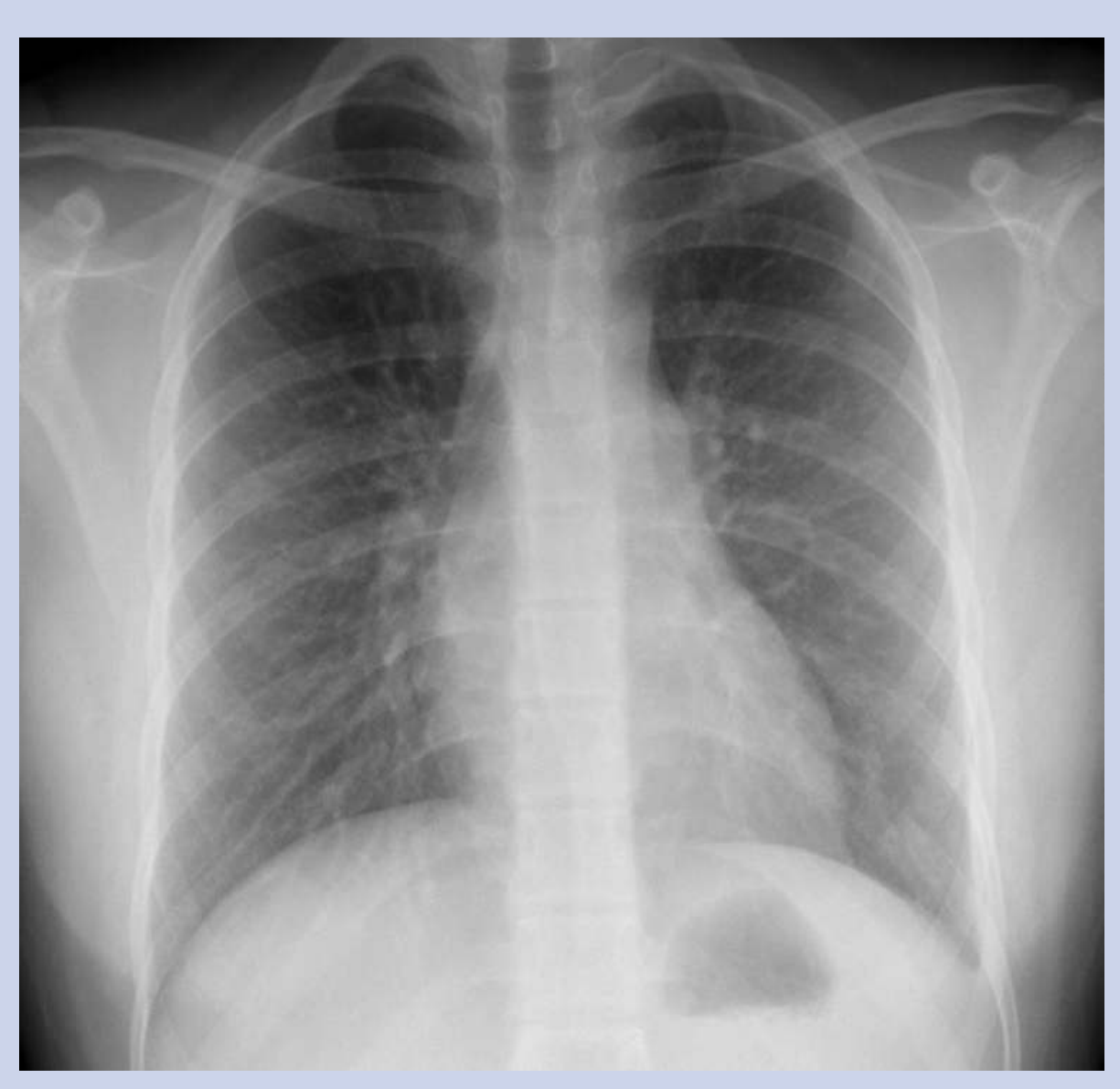

